\title{
Instability of plane Poiseuille flow in a fluid-porous system
}

\author{
Rong Liu, ${ }^{\text {a) }}$ Qiu Sheng Liu, ${ }^{\text {b) }}$ and Si Cheng Zhao \\ National Microgravity Laboratory, Institute of Mechanics, Chinese Academy of Sciences, \\ Beijing 100080, China
}

(Received 9 January 2008; accepted 24 September 2008; published online 20 October 2008)

\begin{abstract}
The instability of Poiseuille flow in a fluid-porous system is investigated. The system consists of a fluid layer overlying porous media and is subjected to a horizontal plane Poiseuille flow. We use Brinkman's model instead of Darcy's law to describe the porous layer. The eigenvalue problem is solved by means of a Chebyshev collocation method. We study the influence of the depth ratio $\hat{d}$ and the Darcy number $\delta$ on the instability of the system. We compare systematically the instability of Brinkman's model with the results of Darcy's model. Our results show that no satisfactory agreement between Brinkman's model and Darcy's model is obtained for the instability of a fluid-porous system. We also examine the instability of Darcy's model. A particular comparison with early work is made. We find that a multivalued region may present in the $(k, \mathrm{Re})$ plane, which was neglected in previous work. Here $k$ is the dimensionless wavenumber and Re is the Reynolds number. (c) 2008 American Institute of Physics. [DOI: 10.1063/1.3000643]
\end{abstract}

\section{INTRODUCTION}

Parallel flow occurs in a wide range of industrial processes and natural phenomena. The instability of parallel flows in particular Poiseuille flow has been extensively investigated for many years. Reynolds studied the instability of Poiseuille flow of water in a pipe in experiments at the end of the 19th century. Further theories of the instability of pipe flow have been developed by many investigators. ${ }^{1,2}$ Thomas $^{3}$ studied the problem of plane Poiseuille flow by solving an eigenvalue problem for the Orr-Sommerfeld equation. Grosch and Salwen ${ }^{4}$ studied the instability of steady and time-dependent plane Poiseuille flow using a set of orthogonal functions. Orszag ${ }^{5}$ proposed a so-called Chebyshev-Tau method to solve numerically the Orr-Sommerfeld equation. His results show that the expansions in Chebyshev polynomials are better suited to the solution of eigenvalue problems than expansions in other orthogonal functions.

Simultaneous flow through both a pure fluid and a porous medium has numerous industrial applications. In previous works, much attention was given to the instability of flow in a porous-fluid system concerned about the thermal convection problem. ${ }^{6-8}$ Nevertheless, the instabilities of Poiseuille flow in a porous-fluid system have, as yet, received little theoretical or computational treatment. To our knowledge, only Chang et al. ${ }^{9}$ have reported calculations of the instability of Poiseuille flow in a porous-fluid system.

Studies of convection in porous medium can be divided into two groups. The single-domain models use a Brinkman formulation for both the liquid and the porous medium. The multiple-domain models use independent sets of equations for the fluid and the porous medium.

A classical description of a fluid-porous system consists of the Navier-Stokes equations in the fluid and Darcy's

\footnotetext{
$\overline{{ }^{a}}$ Electronic mail: liurong@imech.ac.cn.

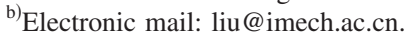

equation in the porous medium. For Darcy's model, clearly defining relevant boundary conditions at the porous-fluid interface remains an open question. In Darcy's model, the viscosity in the porous medium is completely neglected. In many previous works, the tangential velocity component and the tangential shear stress are considered to be discontinuous. Beavers and Joseph ${ }^{10}$ proposed a relation to describe the interfacial velocity given by

$$
\frac{\partial u}{\partial z}=\frac{\alpha}{\sqrt{K}}\left(u-u_{m}\right)
$$

In Eq. (1), $z$ is the direction perpendicular to the interface, $u$ and $u_{m}$ are the horizontal velocities of the fluid and the porous medium, respectively, $K$ is the permeability, and $\alpha$ is the Beavers-Joseph constant which is determined by experiment and varies for different fluids and porous media. This boundary condition was verified experimentally by Beavers and Joseph for a Poiseuille flow over porous media.

A single-domain equation, i.e., the Brinkman equation, has been widely used to describe the flows in the porous layer. A concise derivation of the Brinkman equation can be found in the work of Bars and Worster. ${ }^{11}$ In Brinkman's model, the "effective" viscosity is introduced to describe the friction caused by macroscopic shear. Using this equation implies that all fields are continuous through the whole domain. This approach seems to eliminate the need for defining complicated boundary conditions at the porous-fluid interface.

Nield and Bejan ${ }^{12}$ concluded that for many practical purposes there is no need to include the Laplace term in the Brinkman equation. We note that, in most of the studies, the Darcy equation together with the Beavers-Joseph condition was justified in the basic state. The mass flux was chosen as the criterion. It seems that there is no problem when using the Beavers-Joseph condition for steady Poiseuille flow. Beavers and Joseph ${ }^{10}$ concluded that replacing the effect of 
the boundary layer with a slip velocity proportional to the exterior velocity gradient is shown to be reasonable agreement with experiment results. Nield and Bejan ${ }^{12}$ pointed out that if it is important that a no-slip boundary condition be satisfied, then the Laplacian term is required. In our problem, we are interested in the instability of Poiseuille flow. Since the boundary layer at the interface may influence the instability behavior of the system, the following question should be asked. Even though the Beavers-Joseph condition is valid for the basic Poiseuille flow, could we obtain the same instability behavior if replacing the effect of the boundary layer with a slip velocity? If yes, it indicates that the BeaversJoseph condition is valid for both basic state and the perturbed state. If no, it shows that the influence of boundary layer is important for the instability behavior and the no-slip condition should be retained for instability problems.

In most of the works on the instability of a fluid-porous system, the porous medium is described using Darcy's model. At present, only a few analytical and numerical results on the instability of a fluid-porous system are reported in the framework of Brinkman's model. To our knowledge, Desaive and Lebon ${ }^{8}$ have used Brinkman's model to investigate the instability of thermal convection in a fluid-porous system. They reported that Brinkman's model gives qualitatively the same results as Darcy's law. Zhao and Chen ${ }^{13}$ investigated the stability of double-diffusive convection in superposed fluid and porous layer using Brinkman's model. Hennenberg et al. ${ }^{14}$ developed a tentative study on the Bénard-Marangoni problem using Brinkman's model.

For the instability problem of a fluid-porous system subjected to a plane Poiseuille flow, the following question should be asked. If one can obtain good agreements between Darcy's model and Brinkman's model? We suggest that the viscosity should be taken into account when the effect of viscosity is important, especially in the low permeability case or for the system being subjected to a shear flow. So, in this paper we chose Brinkman's model instead of Darcy's law to describe the porous medium.

The aim of this study is threefold. First, we check carefully the results of Darcy's model reported by previous works. Special emphasis is given to the multivalued region in the $(k, \operatorname{Re})$ plane, which has not been reported by previous works. Second, we use an alternative model, i.e., Brinkman's model, to describe the instability problem for a fluid-porous system. We focus on the influences of the depth ratio and the Darcy number on the instability of the system. Finally, similarities and differences between the characteristics of Brinkman's model and Darcy's model are presented.

\section{THE BRINKMAN EQUATION}

The Brinkman equation is an alternative to the Darcy equation and has been widely used to investigate the flows in porous media. However, a careful look at previous publications shows no general agreement regarding its conditions of applicability. Moreover, the forms of the Brinkman equation in different works are not consistent. Brinkman ${ }^{15}$ obtained via a self-consistent approach the momentum balance equation as

$$
\rho c_{a} \frac{\partial \mathbf{u}}{\partial t}=-\nabla p-\frac{\mu}{K} \mathbf{u}+\mu_{e} \nabla^{2} \mathbf{u},
$$

where $\rho$ is the density of the fluid, $\mu_{e}$ is the effective viscosity, $c_{a}$ is the acceleration coefficient, $\mathbf{u}$ is the Darcy velocity, $p$ is the pressure, $\mu$ is the fluid viscosity, and $K$ is the permeability. Some early investigators ${ }^{12}$ considered that this equation is not simply an extension of the phenomenological Darcy law and is valid for a rather high porosity. Taking for granted Boussinesq's approximation, Desaive and Lebon ${ }^{8}$ used another form to investigate the thermal convection in a fluid-porous system,

$$
\begin{aligned}
\frac{\rho}{\phi} \frac{\partial \mathbf{u}}{\partial t}+\frac{\rho}{\phi^{2}} \mathbf{u} \cdot \nabla \mathbf{u}= & -\nabla p-\rho \mathbf{g}\left[1-\alpha_{T}\left(T-T_{0}\right)\right]-\frac{\mu}{K} \mathbf{u} \\
& +\mu_{e} \nabla^{2} \mathbf{u} .
\end{aligned}
$$

Here $\phi$ is the porosity, $T$ is the temperature of the fluid in porous media, $T_{0}$ is the reference temperature, $\alpha_{T}$ is the constant coefficient of volumic expansion, and $\mathbf{g}$ is the acceleration of gravity. This equation is analogous to the NavierStokes equation and has the $\mathbf{u} \cdot \nabla \mathbf{u}$ term. For the Darcy equation, this term is inconsistent with the slip boundary condition. Moreover, there are further fundamental objections to this term. ${ }^{12}$ Hence, this term is generally dropped in many works. Nield suggested that this term needs to be retained in the case of highly porous media.

Recently, Bars and Worster derived the Brinkman equation based on the volume-averaging method. In their work, they have not used the assumption that the porosity is large and the form of the Brinkman equation in their work is different from those in some early works such as Eqs. (2) and (3). The Brinkman equation in their work is written in our notation as

$$
\frac{\rho}{\phi} \frac{\partial \mathbf{u}}{\partial t}+\rho \frac{\mathbf{u}}{\phi} \cdot \nabla \frac{\mathbf{u}}{\phi}=-\nabla p-\frac{\mu}{K} \mathbf{u}+\frac{\mu_{e}}{\phi} \nabla^{2} \mathbf{u}+\rho \mathbf{g} .
$$

The effective viscosity is defined as

$$
\left[\mu \nabla \mathbf{u}_{l}\right]=\mu_{e} \nabla\left[\mathbf{u}_{l}\right] .
$$

Here [ ] denotes the volume average of a given quantity of liquid per unit volume. The Darcy velocity $\mathbf{u}$ is defined as

$$
\mathbf{u}=\left[\mathbf{u}_{l}\right] \text {. }
$$

In their work, the effective viscosity $\mu_{e}$ is considered to have the same value as $\mu$. The term $\left(\mu_{e} / \phi\right) \nabla^{2} \mathbf{u}$ in Eq. (4) is different from $\mu_{e} \nabla^{2} \mathbf{u}$ in Eq. (3). When $\phi$ is close to unity, Eq. (4) is consistent with Eq. (3).

We must point out that even though some early authors concluded that Brinkman's equation could be valid only when the porosity is sufficiently large, $\phi \geq 0.85$ or more, Bars and Worster ${ }^{11}$ have not limited this equation to highly porous media. In their work, they used this model for $\phi$ $=0.2$ to study corner flow in a fluid overlying a porous layer with constant porosity and for $\phi=0.5$ to study the Poiseuille flow overlying a porous layer. Desaive and Lebon ${ }^{8}$ used the Brinkman model for $\phi=0.3$ to study the thermal convection in a liquid overly a porous layer. Zhao and Chen ${ }^{13}$ studied the double-diffusive convection in superposed fluid and po- 
rous layer for $\phi=0.39$ using the Brinkman equation. Their results show that the Brinkman model and the Darcy model predict the same general characteristics of the marginal curves, and there are differences in the critical conditions. Carefully conducted experiments are needed to determine which model gives the more realistic results.

In this paper, we develop a tentative study on the instability of Poiseuille flow in a fluid overlying a porous layer using the Brinkman model, even though we are fully aware that previous publications show no general agreement regarding its conditions of applicability.

\section{PROBLEM FORMULATION}

We consider an incompressible fluid layer of thickness $d$ overlying a porous layer of thickness $d_{m}$. The interface between the porous medium and the fluid is located at $z=0$. For Poiseuille flow we assume a constant pressure gradient in the $x$ direction. For the instability of Poiseuille flow, Squire's theorem ${ }^{16}$ can reduce the three-dimensional controlling equations into an equivalent two-dimensional counterpart. So, in this paper we only take into account the instability dominated by two-dimensional disturbances.

\section{A. Governing equations and boundary conditions}

The controlling equations in the fluid are the continuity and momentum equations,

$$
\begin{aligned}
& \frac{\partial u}{\partial x}+\frac{\partial w}{\partial z}=0, \\
& \frac{\partial u}{\partial t}+u \frac{\partial u}{\partial x}+w \frac{\partial u}{\partial z}=-\frac{1}{\rho} \frac{\partial p}{\partial x}+\nu \nabla^{2} u, \\
& \frac{\partial w}{\partial t}+u \frac{\partial w}{\partial x}+w \frac{\partial w}{\partial z}=-\frac{1}{\rho} \frac{\partial p}{\partial z}+\nu \nabla^{2} w .
\end{aligned}
$$

In these equations, $t$ is the time, $u$ and $w$ are the horizontal and vertical components of velocity, $p$ is the pressure, $\rho$ is the density of fluid, and $\nu$ is the kinematic viscosity of fluid.

Although Darcy's model is widely used to describe porous medium, the friction caused by macroscopic shear is neglected in this model. It should be noted that the influence of viscous interaction is important at the porous-fluid interface or when the porosity is large. So, we prefer to choose the Brinkman model to describe the porous media. The continuity momentum equations for the porous layer are given by

$$
\begin{aligned}
& \frac{\partial u_{m}}{\partial x}+\frac{\partial w_{m}}{\partial z}=0, \\
& \frac{1}{\phi} \frac{\partial u_{m}}{\partial t}=-\frac{1}{\rho} \frac{\partial p_{m}}{\partial x}+\frac{\mu_{e}}{\rho \phi} \nabla^{2} u_{m}-\frac{\nu_{l}}{K} u_{m}, \\
& \frac{1}{\phi} \frac{\partial w_{m}}{\partial t}=-\frac{1}{\rho} \frac{\partial p_{m}}{\partial z}+\frac{\mu_{e}}{\rho \phi} \nabla^{2} w_{m}-\frac{\nu_{l}}{K} w_{m} .
\end{aligned}
$$

In these equations, $u_{m}$ and $w_{m}$ are the horizontal and vertical components of the pore averaged velocity, $p_{m}$ is the pore averaged pressure, $\phi$ is the porosity, $\mu_{e}$ is the effective viscosity, $K$ is the permeability, and the subscript $m$ denotes porous medium. We should point out that the momentum equation for the porous layer in our analysis is in the different form to some early works in which the Laplacian term is $\left(\mu_{e} / \rho\right) \nabla^{2} u_{m}$. This form of the Brinkman equation is the same as that used in Refs. 13 and 11, in which $\mu_{e}=\mu$. Most investigators suggested that $\mathbf{u}_{m} \cdot \nabla \mathbf{u}_{m}$ items should be dropped except for the case of highly porous media, so in this paper, we have dropped these items in Eqs. (11) and (12).

Upper and lower boundaries are assumed to be rigid walls, at $z=d$ the boundary conditions are

$u=w=0$,

at $z=-d_{m}$

$$
u_{m}=w_{m}=0 .
$$

Many types of interfacial conditions have been used in previous works, however, the definition of boundary conditions at the fluid-porous interface remains an open question. Continuity of normal velocity is robust and generally accepted by most investigators. Until now, it is still an argument that the pressure or the total normal stress is continuous across the interface. In Nield's work, ${ }^{12}$ it is pointed out that in practice, the viscous term may be small compared with the pressure, so the continuity of total normal stress reduces to the approximate continuity of pressure. Chen and Chen, ${ }^{6}$ Bars and Worster, ${ }^{11}$ and Chang et al. ${ }^{9}$ used the continuity of pressure. Desaive and Lebon ${ }^{8}$ and Chang $^{17,18}$ used the continuity of normal stress.

Since the Brinkman model introduces a viscous term analogous to Navier-Stokes equation, we consider it is reasonable that the tangential stress is continuous across the interface, as used in some previous works. ${ }^{8,14}$

In this paper, we used the same boundary conditions as in Desaive's work. ${ }^{8}$ We should point out that in the case of $\mu_{e}=\mu$, the continuity of pressure is the consequence of the continuity of total normal stress. At the porous-fluid interface, interfacial conditions express the continuity of the tangential and normal components of velocity and stress tensor,

$$
\begin{aligned}
& u=u_{m}, \quad w=w_{m}, \\
& \mu \frac{\partial u}{\partial z}=\mu_{e} \frac{\partial u_{m}}{\partial z}, \\
& p-2 \mu \frac{\partial w}{\partial z}=p_{m}-2 \mu_{e} \frac{\partial w_{m}}{\partial z} .
\end{aligned}
$$

\section{B. Unperturbed state solution of the system}

We assume a constant pressure gradient in the $x$-direction. For the fluid layer, we choose the scale of length to be $d$, time to be $d^{2} / \nu$, velocity to be $\nu / d$, and pressure to be $\mu \nu / d^{2}$. For the porous layer, we choose $d_{m}, d_{m}^{2} / \nu, \nu / d_{m}$, and $\mu \nu / d_{m}^{2}$ for the corresponding scales. The dimensionless controlling equations for the horizontal velocities of basic state are 


$$
\frac{d^{2} \bar{U}}{d z^{2}}=\frac{d \bar{P}}{d x}
$$

$$
\frac{\Lambda}{\phi} \frac{d^{2} \bar{U}_{m}}{d z_{m}^{2}}-\delta^{-2} \bar{U}_{m}=\frac{1}{\hat{d}^{3}} \frac{d \bar{P}}{d x} .
$$

At $z_{m}=-1$,

$$
\bar{U}_{m}=0 .
$$

At $z=1$,

$$
\bar{U}=0 .
$$

At $z=z_{m}=0$,

$$
\bar{U}=\hat{d} \bar{U}_{m},
$$

$$
\frac{d \bar{U}}{d z}=\Lambda \hat{d}^{2} \frac{d \bar{U}_{m}}{d z_{m}} .
$$

In these equations, $x$ and $z$ are the dimensionless coordinates for the fluid layer, $x_{m}$ and $z_{m}$ are the dimensionless coordinates for the porous layer, and $\bar{U}$ and $\bar{P}$ denote the horizontal velocity and the pressure of basic state. Re is the Reynolds number defined as $\operatorname{Re}=u_{\max } d / \nu, \delta$ is the Darcy number defined as $\delta=\sqrt{K} / d_{m}, \hat{d}$ is the depth ratio defined as $\hat{d}=d / d_{m}$, and $\Lambda$ is the dynamic viscosity ratio defined as $\Lambda$ $=\mu_{e} / \mu$. Here $u_{\max }$ denotes the maximum horizontal velocity in the fluid. We set the dimensionless maximum velocity in the fluid layer to be 1 , so that the corresponding Reynolds number $u_{\max } d / \nu$ is 1 . If we use $\bar{U}(z)$ and $\bar{U}_{m}(z)$ to denote the velocities for $\operatorname{Re}=1$, the velocity distributions in the liquid and the porous layers are $\operatorname{Re} \bar{U}(z)$ and $\operatorname{Re} \bar{U}_{m}(z)$.

For Darcy's model, the basic flow is uniform in porous medium, ${ }^{9}$

$$
u_{m}=-\frac{K}{\mu} \frac{d p}{d x} .
$$

Because the basic flow will significantly influence the instability of the system, it is helpful and important to compare the basic flow of Brinkman's model with that of Darcy's model.

The basic velocity profiles of Darcy's model for different Darcy numbers are presented in Fig. 1. In the low permeability case $\delta=0.001$, the velocity is symmetric in the fluid layer. For $\delta=0.01$ and 0.1 , the presence of discontinuity of horizontal velocity at the interface significantly breaks the symmetry of velocity in the fluid layer. For Brinkman's model, the basic velocity profiles are shown in Fig. 2. For $\delta$ $=0.001$, the flow is confined in the fluid layer, and the velocity profile of Brinkman's model shows no difference to that of Darcy's model. For $\delta=0.01$ and 0.1 , in the fluid layer the velocity fields of Brinkman's model are more approximate to symmetry than that of Darcy's model.

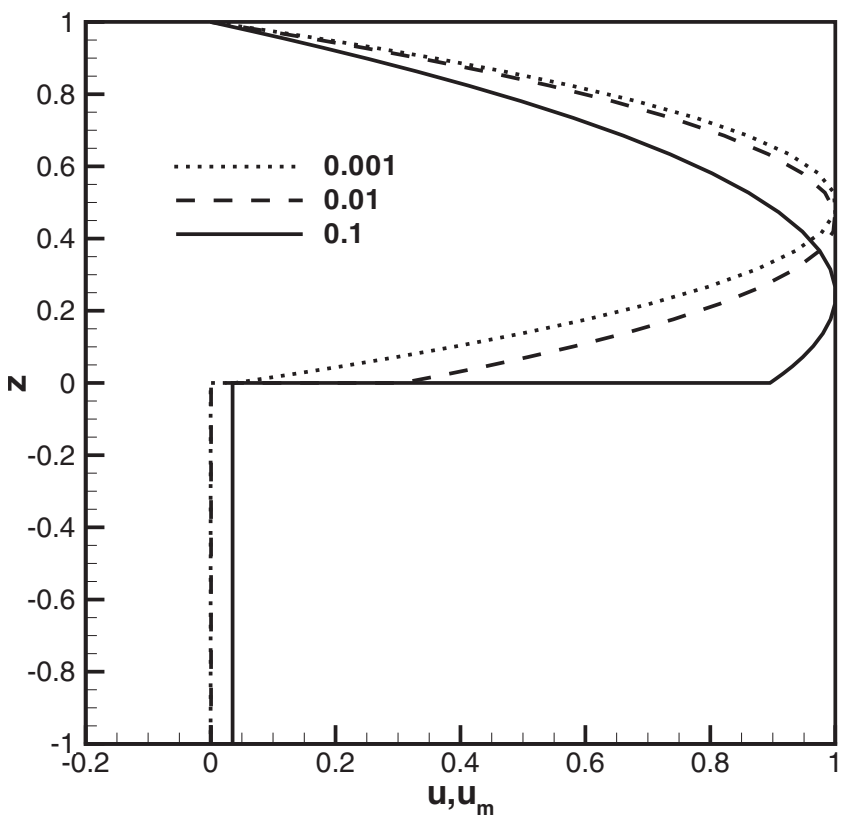

FIG. 1. The basic states of normalized horizontal velocities in both fluid and porous layers for various Darcy numbers $\delta=0.001,0.01$, and 0.1 using Darcy's model. The other parameters are $\phi=0.3, \hat{d}=1.0$, and $\alpha=0.1$.

\section{Perturbation equations}

With the $\mathbf{u}_{m} \cdot \nabla \mathbf{u}_{m}$ items dropped, the dimensionless linearized perturbation equations are

$$
\begin{aligned}
& \frac{\partial u}{\partial x}+\frac{\partial w}{\partial z}=0, \\
& \frac{\partial u}{\partial t}+\operatorname{Re}\left(\bar{U} \frac{\partial u}{\partial x}+\frac{d \bar{U}}{d z} w\right)=-\frac{\partial p}{\partial x}+\nabla^{2} u,
\end{aligned}
$$

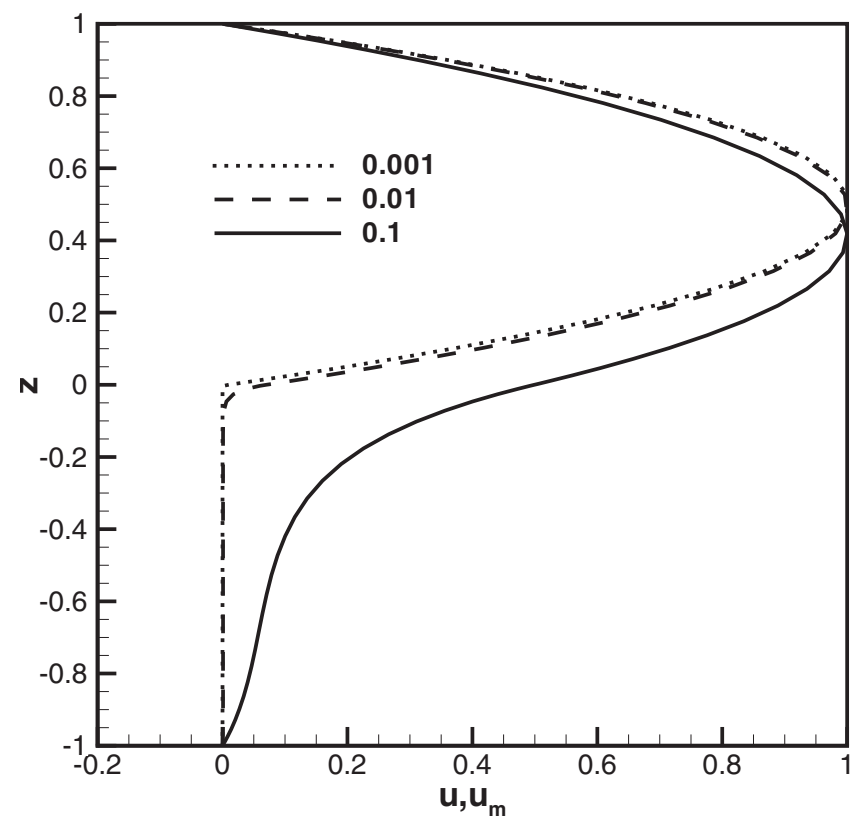

FIG. 2. The basic states of normalized horizontal velocities in both fluid and porous layers for various Darcy numbers $\delta=0.001,0.01$, and 0.1 using Brinkman's model. The other parameters are $\phi=0.3, \hat{d}=1.0$, and $\Lambda=1.0$. 


$$
\begin{aligned}
& \frac{\partial w}{\partial t}+\operatorname{Re} \bar{U} \frac{\partial w}{\partial x}=-\frac{\partial p}{\partial z}+\nabla^{2} w, \\
& \frac{\partial u_{m}}{\partial x_{m}}+\frac{\partial w_{m}}{\partial z_{m}}=0, \\
& \frac{1}{\phi} \frac{\partial u_{m}}{\partial t_{m}}=-\frac{\partial p_{m}}{\partial x_{m}}+\frac{\Lambda}{\phi} \nabla_{m}^{2} u_{m}-\delta^{-2} u_{m}, \\
& \frac{1}{\phi} \frac{\partial w_{m}}{\partial t_{m}}=-\frac{\partial p_{m}}{\partial z_{m}}+\frac{\Lambda}{\phi} \nabla_{m}^{2} w_{m}-\delta^{-2} w_{m} .
\end{aligned}
$$

At $z=1$,

$$
u=0, \quad w=0 .
$$

At $z_{m}=-1$,

$$
u_{m}=0, \quad w_{m}=0 .
$$

At $z=z_{m}=0$,

$$
\begin{aligned}
& u=\hat{d} u_{m}, \quad w=\hat{d} w_{m} \\
& \frac{\partial u}{\partial z}+\frac{\partial w}{\partial x}=\hat{d}^{2} \Lambda\left(\frac{\partial u_{m}}{\partial z_{m}}+\frac{\partial w_{m}}{\partial x_{m}}\right), \\
& p-2 \frac{\partial w}{\partial z}=\hat{d}^{2}\left(p_{m}-2 \Lambda \frac{\partial w_{m}}{\partial z_{m}}\right) .
\end{aligned}
$$

We introduce the normal modes

$$
\begin{aligned}
(u, w, p)=[ & U(z), W(z), P(z)] \exp (\lambda t+i k x), \\
\left(u_{m}, w_{m}, p_{m}\right)= & {\left[U_{m}\left(z_{m}\right), W_{m}\left(z_{m}\right), P_{m}\left(z_{m}\right)\right] } \\
& \times \exp \left(\lambda_{m} t_{m}+i k_{m} x_{m}\right) .
\end{aligned}
$$

Here $U(z), W(z), P(z), U_{m}\left(z_{m}\right), W_{m}\left(z_{m}\right)$, and $P_{m}\left(z_{m}\right)$ are the amplitudes of $u, w, p, u_{m}, w_{m}$, and $p_{m}$. $\lambda$ and $\lambda_{m}$ are the time growth rates of the fluid layer and the porous layer and $k$ and $k_{m}$ are the dimensionless wavenumbers of both layers. Note that $k=\hat{d} k_{m}$ and $\lambda=\hat{d}^{2} \lambda_{m}$.

The normal mode equations of the amplitudes are

$$
\begin{aligned}
& i k U+D W=0, \\
& \lambda U+\operatorname{Re}\left(i k \bar{U} U+\bar{U}^{\prime} W\right)=-i k P+\left(D^{2}-k^{2}\right) U, \\
& \lambda W+i k \operatorname{Re} \bar{U} W=-D P+\left(D^{2}-k^{2}\right) W, \\
& i k_{m} U_{m}+D_{m} W_{m}=0, \\
& \frac{1}{\phi} \lambda_{m} U_{m}=-i k_{m} P_{m}+\frac{\Lambda}{\phi}\left(D_{m}^{2}-k_{m}^{2}\right) U_{m}-\delta^{-2} U_{m}, \\
& \frac{1}{\phi} \lambda_{m} W_{m}=-D_{m} P_{m}+\frac{\Lambda}{\phi}\left(D_{m}^{2}-k_{m}^{2}\right) W_{m}-\delta^{-2} W_{m} .
\end{aligned}
$$

At $z=1$,

$$
\begin{aligned}
& U=0, \quad W=0 . \\
& U_{m}=0, \quad W_{m}=0 . \\
& U=\hat{d} U_{m}, \quad W=\hat{d} W_{m} . \\
& D U+i k W=\hat{d}^{2} \Lambda\left(D_{m} U_{m}+i k_{m} W_{m}\right), \\
& P-2 D W=\hat{d}^{2}\left(P_{m}-2 \Lambda D_{m} W_{m}\right) .
\end{aligned}
$$$$
\text { At } z_{m}=-1 \text {, }
$$$$
\text { At } z=z_{m}=0 \text {, }
$$

Here $D$ and $D_{m}$ are the differential operators defined as $d / d z$ and $d / d z_{m}$. Equations (38)-(43) together with the boundary conditions (44)-(48) determine an eigenvalue problem in the form of

$$
F(\lambda, \operatorname{Re}, k, \hat{d}, \phi, \delta, \Lambda)=0 .
$$

\section{NUMERICAL METHOD}

The spectral method can yield great accuracy for the convective instability problems. A wide variety of spectral schemes applied to fluid dynamics have been reviewed in the work by Canuto et al. ${ }^{19}$ We implement a Chebyshev collocation method to solve this eigenvalue problem. We first transform the domains of each layer $(0,1)$ and $(0,-1)$ to the Chebyshev domain $(-1,1)$ by introducing $\eta=2 z-1$ and $\eta_{m}$ $=-2 z_{m}-1$. The variables $U, W, P, U_{m}, W_{m}$, and $P_{m}$ are expanded as Chebyshev series

$$
U=\sum_{n=0}^{N} \widetilde{U}_{n} T_{n}(\eta), \quad W=\sum_{n=0}^{N} \widetilde{W}_{n} T_{n}(\eta), \quad P=\sum_{n=0}^{N} \widetilde{P}_{n} T_{n}(\eta),
$$

$$
\begin{aligned}
& U_{m}=\sum_{n=0}^{N} \widetilde{U}_{n}^{m} T_{n}\left(\eta_{m}\right), \quad W_{m}=\sum_{n=0}^{N} \tilde{W}_{n}^{m} T_{n}\left(\eta_{m}\right), \\
& P_{m}=\sum_{n=0}^{N} \widetilde{P}_{n}^{m} T_{n}\left(\eta_{m}\right) .
\end{aligned}
$$

Here $T_{n}$ are the Chebyshev polynomials defined as

$$
T_{n}(x)=\cos k \theta, \quad \theta=\arccos x, \quad x \in[-1,1] .
$$

A particularly convenient choice for the collocation points $x_{j}$ is

$$
x_{j}=\cos \frac{\pi j}{N} .
$$

Equations (38)-(43) together with corresponding boundary conditions are an eighth order eigenproblem. Using the Chebyshev series (50) and (51), the system of equations is required to solve for $6(N+1)$ unknowns. The details of imposing boundary conditions in the spectral method for collocation method are described in the work of Tuckeman. ${ }^{20}$ For Eqs. (39), (40), (42), and (43), each is applied at $N-1$ internal collocation points, and at two boundary points these 
TABLE I. The wave velocities corresponding to the leading eigenvalues of the time growth rate for $N=30,40,50,60$, and 70 for several assigned values of $\phi=0.8, \delta=0.1, \Lambda=1$ and $\hat{d}=0.2, \operatorname{Re}=20000.0$, and $k=1.0$.

\begin{tabular}{ccc}
\hline \hline$N$ & $c_{r}$ & $c_{i}$ \\
\hline 30 & $0.184636 \times 10^{00}$ & $0.330819 \times 10^{-02}$ \\
40 & $0.184826 \times 10^{00}$ & $0.316574 \times 10^{-02}$ \\
50 & $0.184774 \times 10^{00}$ & $0.312234 \times 10^{-02}$ \\
60 & $0.184782 \times 10^{00}$ & $0.312887 \times 10^{-02}$ \\
60 & $0.184777 \times 10^{00}$ & $0.312828 \times 10^{-02}$ \\
\hline
\end{tabular}

equations are replaced by boundary conditions. We should point out that the continuity equations (38) and (41) are applied at all collocation points; in other words, the divergence of velocity is zero at both the inner and the boundary points. This gives rise to a $6(N+1) \times 6(N+1)$ matrix eigenvalue problem of the form

$$
A X=\lambda B X .
$$

We have checked the convergence of our numerical results. For example, in the high porosity case $\phi=0.8, \delta=0.1, \Lambda=1$, $\hat{d}=0.2, \operatorname{Re}=20000.0$, and $k=1.0$, for $N=30,40,50,60$, and 70 , the wave velocities $c=\lambda / i k$ Re corresponding to the leading eigenvalue $\lambda$ are listed in Table I. $c_{r}$ and $c_{i}$ are the real part and the imaginary part of $c$. It is found that $N=40$ is enough to obtain satisfactory results.

\section{RESULTS AND DISCUSSION}

\section{A. Classical results of Poiseuille flow}

We first check our calculation procedure and program by comparing our results of classical plane Poiseuille flow with the results of previous works. 3,5 A critical comparison between various methods for the solution of plane Poiseuille flow is conveniently made for the most unstable mode with $k=1, \mathrm{Re}=10000$. Orszag used a Chebyshev tau method to solve the Orr-Sommerfeld instability equation. His result is $c=0.23752649+i 0.00373967$. Here $c$ is the wave velocity. We used a Chebyshev collocation method to solve directly the linearized Navier-Stokes equations instead of the OrrSommerfeld equation. Our result is $c=0.23784489$ $+i 0.003753167$. Our result is in good agreement with Orszag's result.

\section{B. Results of Darcy's model}

Chang et al. $^{9}$ first investigated the instability of Poiseuille flow in a fluid-porous system. We will check our calculation by comparing our results of Darcy's model with the results in Ref. 9. In this process, we have uncovered some aspects heretofore overlooked in Chang's work.

Figure 3 presents the marginal curves of the Reynolds number for various depth ratios. Chang et al. ${ }^{9}$ reported three instability modes in detail for lower permeability case $\delta$ $=0.001$. For $\hat{d}=0.11$, the marginal curve displays a bimodal structure and the dominant mode of instability is found in the long wave region. This instability mode is called the porous mode and is characterized by a convection penetrating into

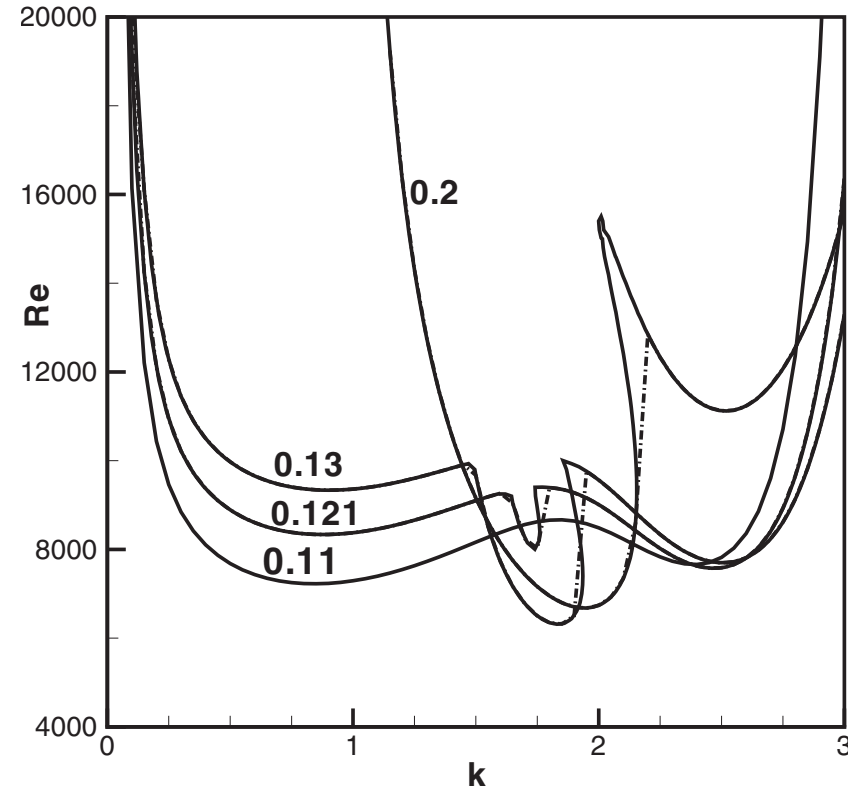

FIG. 3. The marginal curves of the Reynolds number vs the wavenumber for different depth ratios: results of the present study (solid line) and the result of Chang et al. (solid dashed line). The other parameters are $\phi=0.3$, $\delta=0.001$, and $\alpha=0.1$.

the porous medium. For $\hat{d}=0.12$, the instability switches from a long wave mode to a short wave mode. When this short wave mode prevails, most disturbances of the flow are confined in the fluid layer and the real part of the perturbed streamfunction is an odd symmetric function with respect to the centerline of the fluid layer. So, it is called the odd-fluidlayer mode. For $\hat{d}=0.121$, a so-called even-fluid-layer branch occurs in the moderate wave region. The convection of evenfluid-layer mode is largely controlled by the fluid layer and the real part of the streamfunction is approximately symmetric with respect to the centerline of the fluid layer.

The results of the present study (solid lines) are in good agreement with the result of Chang et al. (dot-dashed lines) except in the region between the middle branch and the right branch. In the result of Chang et al. (dot-dashed lines), a straight line connects these two branches. In the present study, the middle branches are multivalued. The multivalued property is neglected in the work of Chang et al., so the straight lines connecting the middle and the right branches are incorrect. It is possible that Chang et al. simply connected the branches and unaware of the possibility of multiple critical value. Fortunately, in general, the minima on the neutral curves are out of the multivalued regions, so unawareness of them will not give incorrect results.

The influence of the Darcy number on the instability was first described by Chang et al. for Darcy's model. Chang et al. computed the marginal curves for $\delta=5 \times 10^{-4}, 8$ $\times 10^{-4}, 1 \times 10^{-3}, 2 \times 10^{-3}, 3 \times 10^{-3}$, and $4 \times 10^{-3}$ in the region of $\operatorname{Re} \leq 20000$. Their results show that for the smaller permeability $(\delta<0.001)$ case the even-fluid-layer (medium wave) mode is dominant, whereas for the larger permeability $(\delta>0.002)$ case the porous (long wave) mode occurs. However, we find that some aspects are overlooked in the results of Chang et al. Here we want to complement the result of 

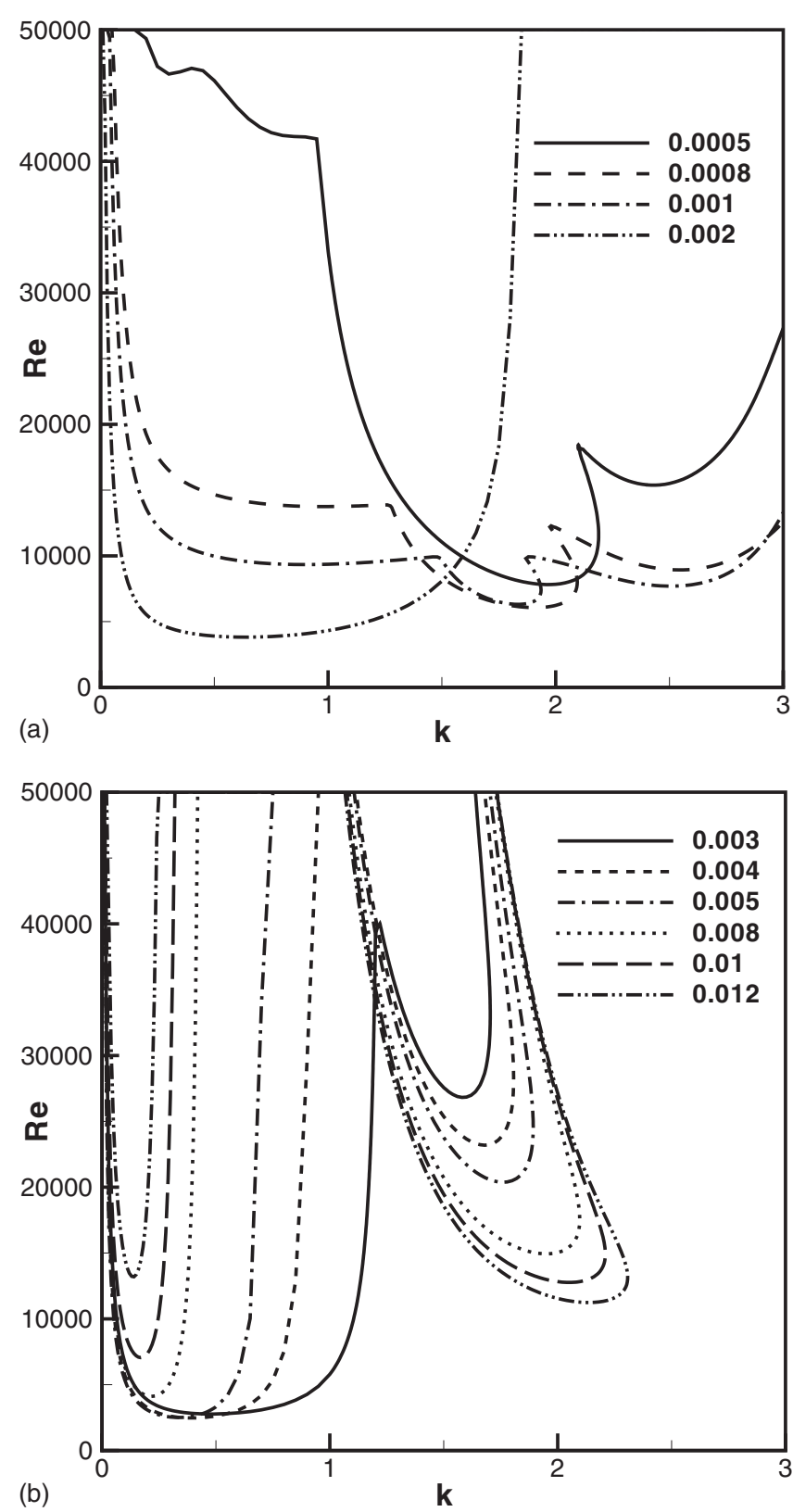

FIG. 4. The marginal curves of the Reynolds number vs the wavenumber for different Darcy numbers. The other parameters are $\phi=0.3, \hat{d}=0.13$, and $\alpha=0.1$.

Chang et al. by examining the instability for a more wide range of Darcy number. In Fig. 4(a), we present the marginal curves for $\delta=5 \times 10^{-4}, 8 \times 10^{-4}, 1 \times 10^{-3}$, and $2 \times 10^{-3}$. The marginal curves in Fig. 4(a) are in good agreement with the result of Chang et al. except for the multivalued region. In Fig. 4(b), we present the marginal curves for $\delta=3 \times 10^{-3}, 4$ $\times 10^{-3}, 5 \times 10^{-3}, 8 \times 10^{-3}, 1 \times 10^{-2}$, and $1.2 \times 10^{-2}$. In Fig. 4(b), all curves display a bimodal structure. For $\delta=3 \times 10^{-3}$, the long wave mode is dominant. With the increase in $\delta$, the right branch becomes more unstable and the left branch becomes more stable. For $\delta=1.2 \times 10^{-2}$, the short wave mode becomes dominant. These results indicate that the influence of $\delta$ on the instability mode is somewhat complicated. In the small permeability case the even-fluid-layer mode is dominant, and with the increase in $\delta$ the long wave mode becomes more unstable. With even larger $\delta$, a new branch enters in the short wave region and becomes the dominant mode. We note that the right branch for $\delta>0.002$ was overlooked by Chang et al, therefore their result indicates that the long wave mode occurs for large $\delta$.

\section{Comparison with results of Darcy's law}

In the limit $\delta \rightarrow 0$, if we omit the nonlinear item, the Brinkman equation reduces to the Darcy equation

$$
\frac{1}{\phi} \frac{\partial \mathbf{u}}{\partial t}=-\frac{1}{\rho} \nabla p-\frac{\nu}{K} \mathbf{u} .
$$

Note that the Darcy equation is a first order equation, and the Brinkman equation is a second order equation. The controlling equations for a fluid-porous system is sixth order for Darcy's model and eighth order for Brinkman's model. At the bottom, because the viscosity is overlooked in Darcy's model, the bottom boundary has to be regarded as a slip wall. For the Darcy approach, the continuities of the horizontal velocity and the horizontal stress are substituted by the Beavers and Joseph condition, i.e., Eq. (1), at the interface. According to Eq. (1), a larger $\alpha$ corresponds to a larger velocity gradient or shear stress. For a smaller $\alpha$, the shear stress at the interface is smaller. The dimensionless number $\alpha$ has various values for each particular system, and it will significantly influence the instability of the system. Chang et al. ${ }^{9}$ investigated the effects of $\alpha$ on the instability of the system. We first present the marginal curves of Darcy's model for different $\alpha$ and then compare them with the result of Brinkman's model.

\section{The instability of onset of convection}

In Fig. 5, for $\alpha=0.08$, the marginal curve is bimodal. For $\alpha=0.1$, the marginal curve gets more unstable and a new branch presents in the moderate wave region. Such a new branch corresponds to the even-fluid-layer mode in the work of Chang et al. ${ }^{9}$ With the increase in $\alpha$, the even-fluid-layer mode becomes dominant. In this figure, the marginal curve of Brinkman's model displays a bimodal structure. The dominant mode of instability is found for $k=2.25$ with $\mathrm{Re}$ $=2802.8$. The minimum in the long wave branch is found for $k=0.5$ with $\mathrm{Re}=10987.1$.

In order to understand the physics of the two instability mode of Brinkman's model, we present the eigenfunction $W$ in Fig. 6. Figure 6(a) shows the eigenfunction $W$ corresponding to the most unstable mode in the long wave branch. In this figure, the flow penetrates into the porous layer and a reverse flow presents near the fluid-porous interface. This long wave mode of Brinkman's model is qualitatively the same as that of Darcy's model. In Fig. 6(b), the eigenfunction $W$ is nearly symmetric with respect to the centerline of the fluid layer. This result confirms that the right branch of Brinkman's mode is the even-fluid-layer branch.

In Fig. 5, it is shown that Brinkman's model predicts quantitatively the same result with Darcy's model in the long wave limit. As we know, the viscosity mainly operates in the short wave region, therefore, the marginal curves of these two models show no difference in the long wave limit. When 


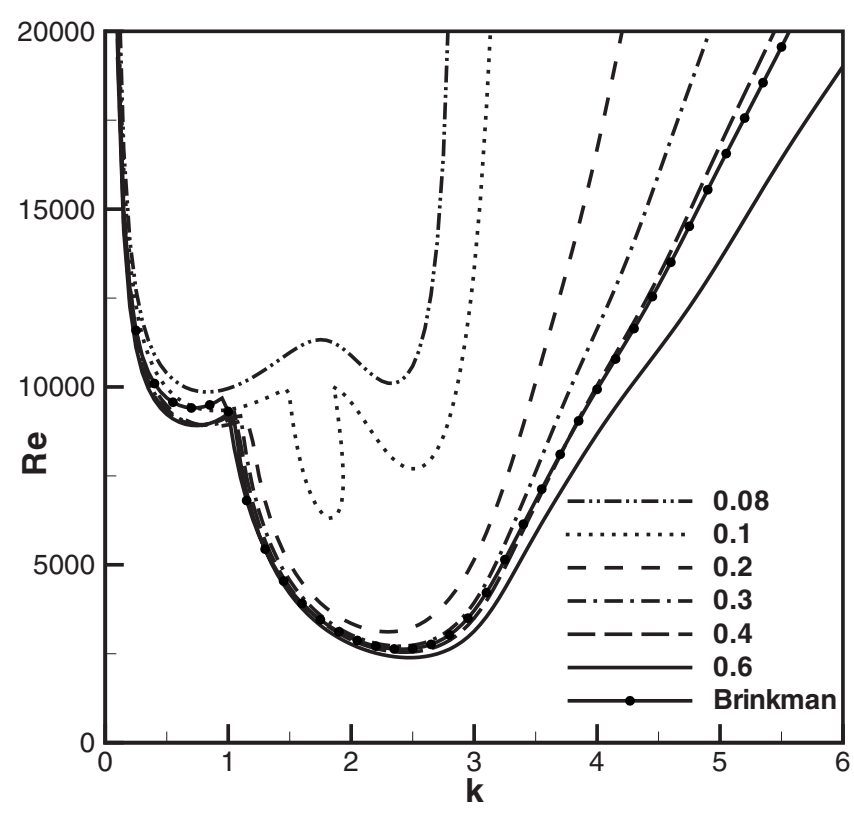

FIG. 5. The marginal curves of the Reynolds number vs the wavenumber using Brinkman's model in comparison with Darcy's model. The other parameters are $\phi=0.3, \hat{d}=0.13, \Lambda=1.0, \delta=0.001$, and $\alpha=0.08,0.1,0.2,0.3$, 0.4 , and 0.6 .

the long wave mode instability occurs, the convection occurs in both the fluid and the porous medium. For Brinkman's model, boundary layers exist near the interface and the bottom wall. However, the presence of boundary layer almost has no influence on the long wave mode instability.

In the moderate wave region, the instability of Brinkman's model is obviously different to the case of smaller $\alpha$. When $\alpha \geq 0.2$, the curves of $\alpha=0.2,0.3,0.4$, and 0.6 are quantitatively similar to that of the Brinkman model. This result indicates that the interfacial shear stress plays an important role in determining the instability. According to Eq. (1), the Beavers-Joseph condition, a larger $\alpha$ corresponds to a larger interfacial stress or a smaller interfacial velocity discontinuity. It is easy to understand that $u_{m}$ is small in a low permeability porous medium. Let us consider the limit case of $u-u_{m}=0$ at the interface. For Darcy's model, a large $\alpha$ and a small $\delta$ can lead to a small $u$; for Brinkman's model, a small $\delta$ ensures the velocity $u$ to be small. When $u \rightarrow 0$ at the interface, both the basic flow and the perturbed flow are symmetric with respect to the centerline of the fluid layer. For a plane Poiseuille flow, the even symmetry of basic flow precludes the possibility of the disturbance of odd-fluid-layer mode. So, in Fig. 5 for Darcy's model, the even-fluid-layer mode becomes dominant and the odd-fluid-layer mode disappears with the increase in $\alpha$; and for Brinkman's model, the odd-fluid-layer mode does not appear.

As shown in Fig. 5, the instability characteristics of $\alpha$ $=0.4$ are very close to that of the Brinkman model in all the wavenumber ranges. For $\alpha \geq 0.2$, the instability characteristics of the Darcy model are close to that of the Brinkman model in the long wave and medium wave ranges. For $\alpha$ $>0.4$ or $<0.4$, in the short wave range the viscous effect of the Darcy model is overestimated or underestimated in comparison with the Brinkman model.
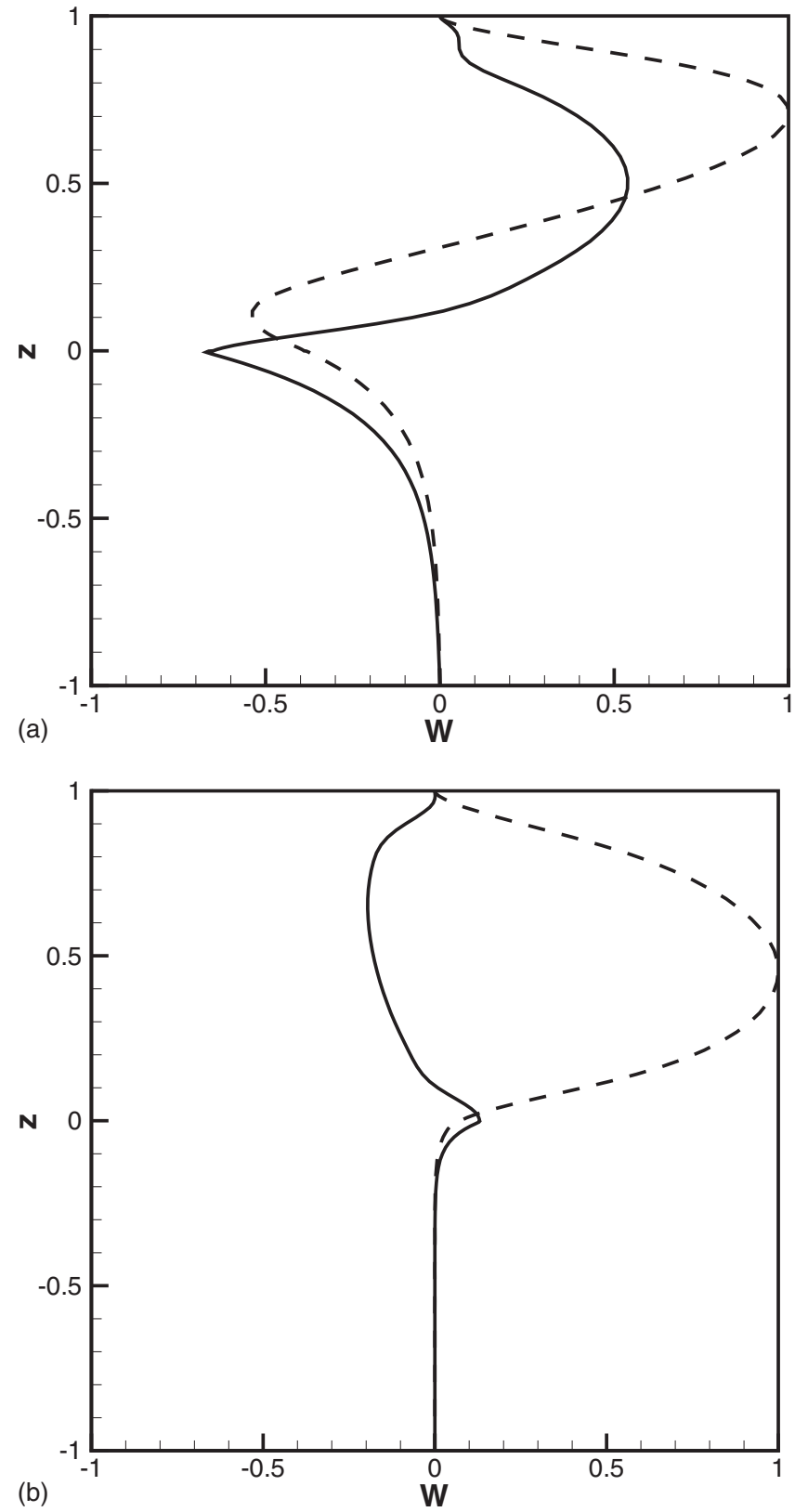

FIG. 6. The amplitude of the vertical velocity $W$ for Brinkman's model. (a) $k=0.7$; (b) $k=2.4$. The solid lines represent the real parts and the dashed lines represent the imaginary parts. The other parameters are $\phi=0.3, \delta$ $=0.001, \Lambda=1.0$, and $\hat{d}=0.13$.

\section{The secondary instability}

For the plane Poiseuille flow in a fluid-porous system, the problem of secondary instability has received little attention in published literature. Moreover, analytical and numerical works are very limited. Here we aim to study the secondary instability within the scope of linear analysis.

First we will describe the characteristics of temporally growing disturbances of Poiseuille flow for Darcy's model. Figure 7 gives curves of constant real part of the time growth rate $\operatorname{Re}(\lambda)$ of the most unstable mode for Darcy's model. It can be seen that in the marginal curve of $\operatorname{Re}(\lambda)=0$, the evenfluid-layer mode (middle branch) is dominant. The critical Reynolds number for the onset of convection is $\mathrm{Re}=6314$ with critical wavenumber $k=1.83$. With the increase in the 


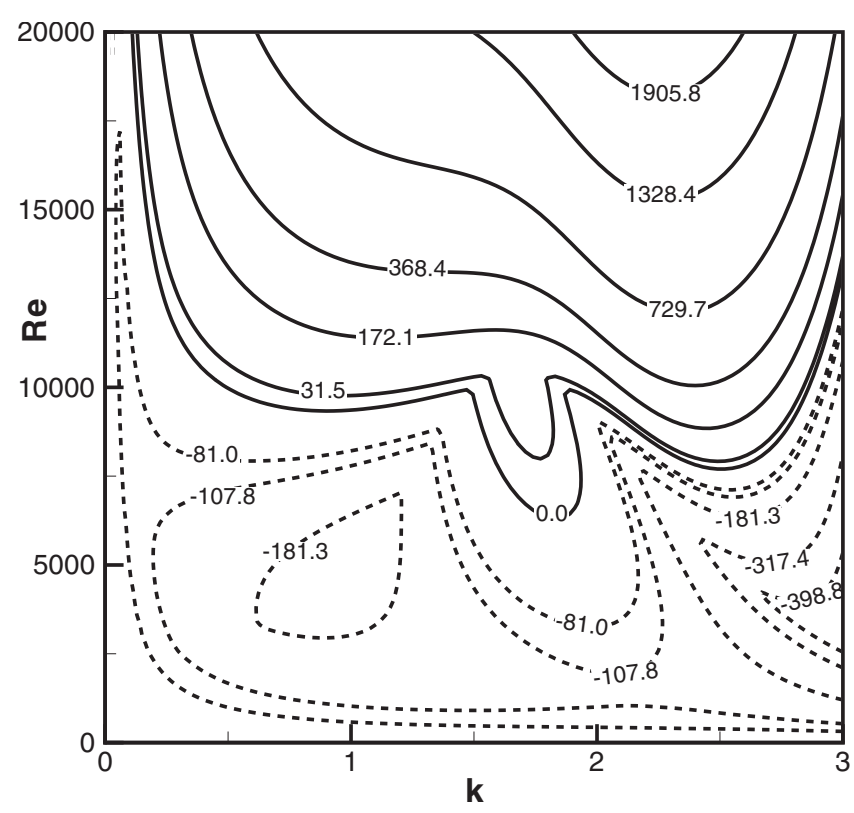

FIG. 7. Curves of constant real part of time growth rate $\lambda$ in the $(k, \operatorname{Re})$ plane for Darcy's model, with $\delta=0.001, \hat{d}=0.13, \alpha=0.1$, and $\phi=0.3$.

Reynolds number, the system becomes more unstable. When the Reynolds number reaches $\operatorname{Re}=7700$, the short wave mode (right branch) reaches the marginal condition $\operatorname{Re}(\lambda)$ $=0$. When $6314<\operatorname{Re} \leq 7700$, only the even-fluid-layer mode is excited. When $\operatorname{Re}=7900$, both the short wave and the even-fluid-layer mode are excited with the time growth rate $\operatorname{Re}(\lambda)=31.5$. In the case of $7700<\operatorname{Re} \leq 7900$, both the evenfluid-layer mode and the short wave mode are excited; however, the latter mode is suppressed by the former mode. When $\operatorname{Re}>7900$, the short wave mode becomes more unstable and suppresses the even-fluid-mode. As discussed above, even-fluid-layer mode occurs on the onset of convection, and with the increase in Reynolds number, the secondary instability is dominated by the short wave (odd-fluidlayer) mode.

Figure 8 gives curves of constant real part of the time growth rate $\operatorname{Re}(\lambda)$ of the most unstable mode for Brinkman's model. It is shown that the even-fluid-layer mode is favored whatever the values of the Reynolds number. In comparison with Darcy's model, no secondary instability has been found for Brinkman's model within the scope of linear analysis. We should note that the secondary instability may be found in the nonlinear evolution. However, the full nonlinear stability analysis or numerical simulation is out of the scope of this paper.

\section{Effect of depth ratio $\hat{d}$}

Figure 9(a) shows the marginal curves for different depth ratios in the low porosity case. For $\phi=0.3, \hat{d}$ $=0.05,0.1,0.12,0.15$ the marginal curves present a bimodal structure. With the increase in $\hat{d}$, both the long wave and the

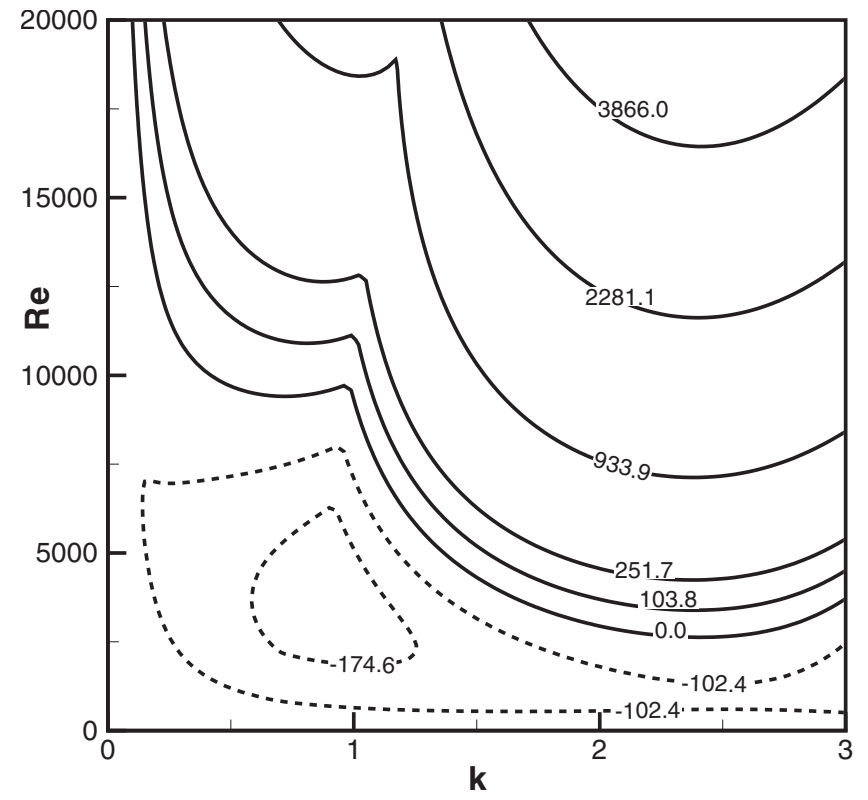

FIG. 8. Curves of constant real part of time growth rate $\lambda$ in the $(k, \operatorname{Re})$ plane for Brinkman's model, with $\delta=0.001, \Lambda=1.0, \hat{d}=0.13$, and $\phi=0.3$.

short wave branches become more stable. It can be seen that the short wave branch predicts a lower critical value, so the even-fluid-layer mode is dominant.

Chang et $a l .^{9}$ have investigated the influence of depth ratio $\hat{d}$ on the stability of a fluid-porous system in the framework of Darcy's model. For low porosity and low permeability $\phi=0.3, \delta=0.001$, their results show that when $\hat{d}$ changes from 0.11 to 0.12 , the instability changes from a porous mode (long wave) to odd-fluid-layer dominated mode (short wave). For $\hat{d}=0.121$, a new instability (even-fluid-layer) mode presents between the long wave branch and the short wave branch. With the increase in $\hat{d}$, the even-fluid-layer mode becomes dominant.

In this study, the influence of $\hat{d}$ on the instability for Brinkman's model is different to Darcy's model. For Brinkman's model with low porosity and low permeability, the even-fluid-layer instability is the dominant mode for different depth ratios. Although the marginal curve may be bimodal, the short wave branch (even-fluid-layer mode) is lower than the long wave branch (porous mode). So, such a transition from a porous mode to an even-fluid-layer mode for Darcy's mode has not been found for Brinkman's model in low porosity and low permeability case.

The instability of high porosity and high permeability fluid-porous system is different from that of low porosity and low permeability. In Fig. 9(b), the marginal curves are shown for different depth ratios $\hat{d}=0.05,0.06,0.07,0.08,0.1,0.12$ with higher porosity $\phi=0.8$ and higher permeability $\delta$ $=0.01$. It is shown that for smaller depth ratios, the long wave mode is preferred. For a larger depth ratio, the evenfluid branch enters at the short wave region. With the increase in the depth ratio, the system becomes more unstable and the short wave mode is preferred. 

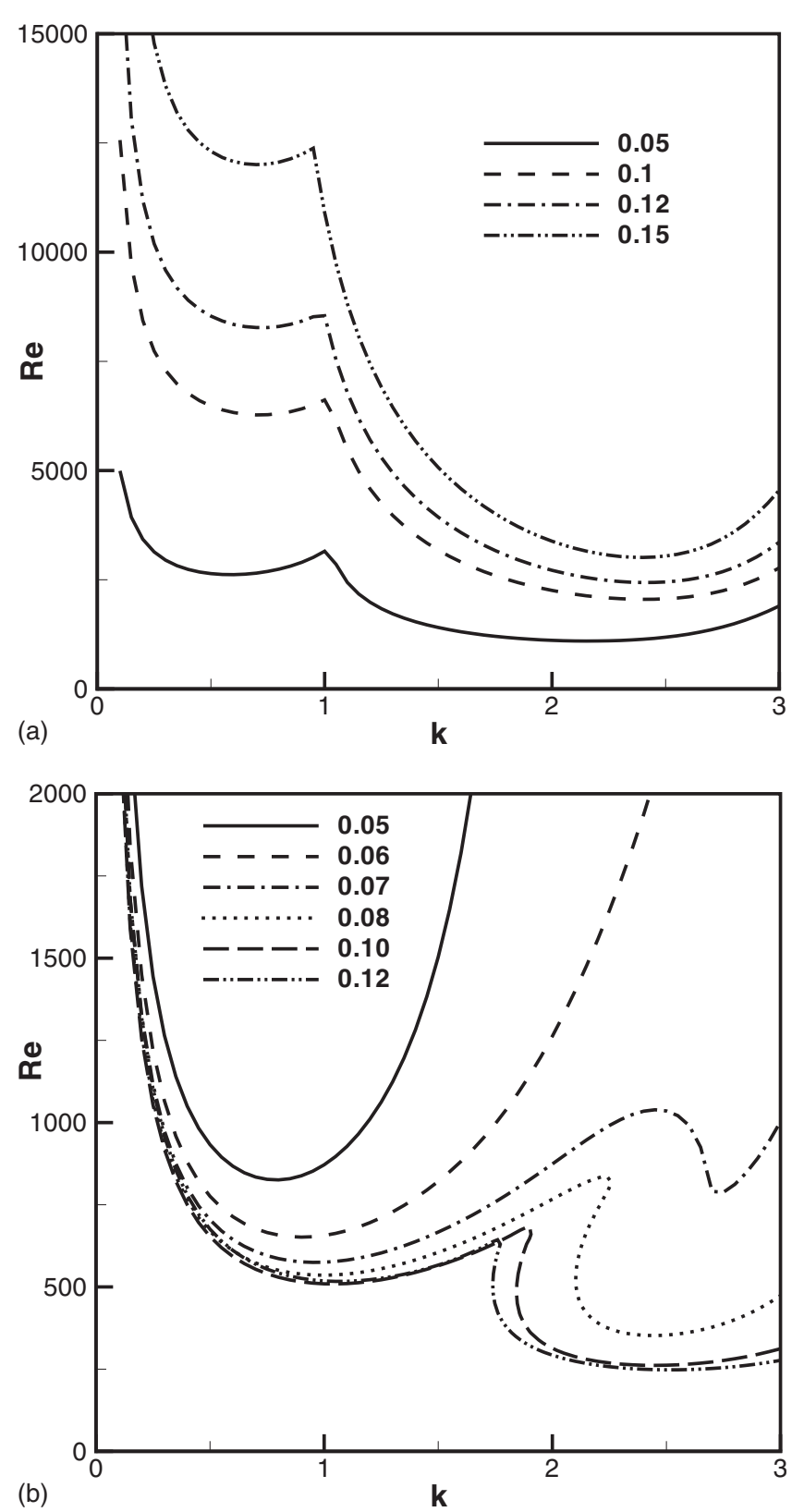

FIG. 9. The marginal curves of the Reynolds number vs the wavenumber for different depth ratios using Brinkman's model. The other parameters are (a) $\phi=0.3, \Lambda=1.0, \delta=0.001$ and (b) $\phi=0.8, \Lambda=1.0, \delta=0.01$.

\section{E. Effect of the Darcy number $\delta$}

The Darcy number $\delta$ is an important parameter to influence the instability of a fluid-porous system. Figure 10 shows the marginal curves for different Darcy numbers $\delta$ with $\hat{d}=0.13$. For $\delta=0.001,0.003,0.005,0.008,0.01,0.012$ each curve exhibits a bimodal structure. For $\delta=0.001$, the long wave branch is more stable than the short wave branch. When $\delta<0.008$, with the increase in $\delta$, both the long wave and the short wave branches become more unstable. But, the short wave branch is lower than the long wave branch, and the even-fluid-layer mode is preferred. When $\delta=0.008$, the minimum in the short wave branch is equal to that in the long wave branch. When $\delta>0.008$, with the increase in $\delta$, the minimum of the long wave branch almost does not

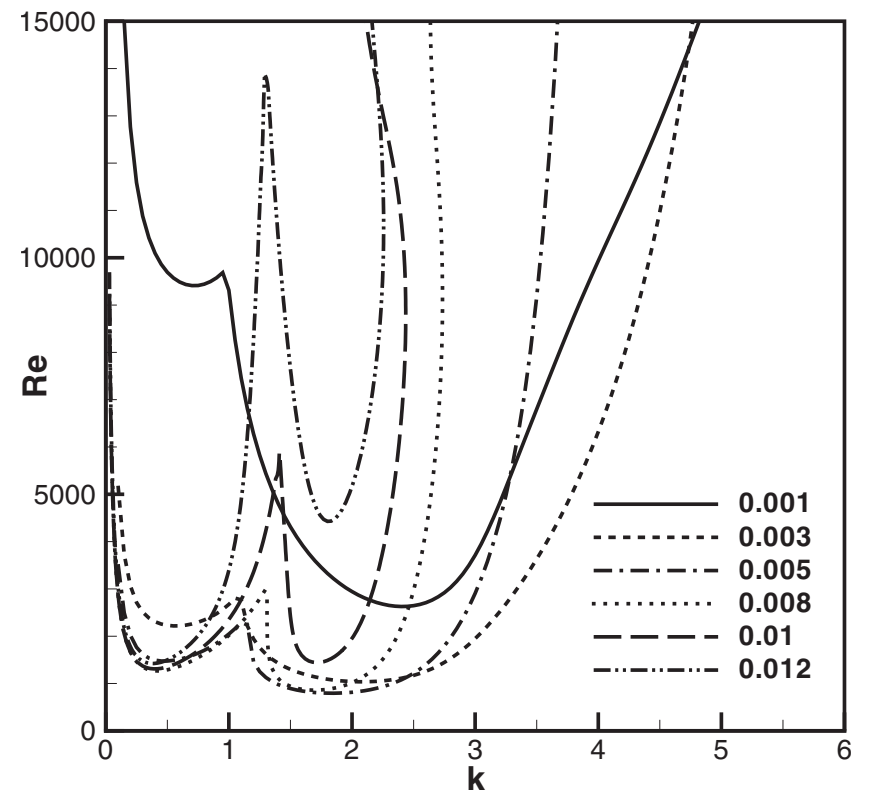

FIG. 10. The marginal curves of the Reynolds number vs the wavenumber for different Darcy numbers $\delta$ using Brinkman's model. The other parameters are $\phi=0.3, \Lambda=1.0$, and $\hat{d}=0.13$.

change, but the short wave branch gets more stable than the long wave branch. As a result, the porous mode suppresses the even-fluid-layer mode.

Chang et $a l .{ }^{9}$ have investigated the influence of the Darcy number $\delta$ on the stability for Darcy's model. They presented the marginal curves for $\delta=5.0 \times 10^{-4}, 8.0 \times 10^{-4}$, $1.0 \times 10^{-3}, 2.0 \times 10^{-3}, 3.0 \times 10^{-3}$, and $4.0 \times 10^{-3}$. These curves are also shown in Fig. 4 in this paper. Their results show that, owing to a larger permeability in the porous layer, the instability can more easily occur in the porous medium. The convection switches from an even-fluid-layer mode to a porous mode in the range of $1.0 \times 10^{-3}<\delta<2.0 \times 10^{-3}$.

For Brinkman's model, as shown in Fig. 10, such a transition from an even-fluid-layer mode to a porous mode has also been found. But such a transition occurs when $\delta$ $=0.008$, which is much higher than the result of Darcy's model.

\section{CONCLUSIONS}

In the present paper, we study the instability of a fluidporous system subjected to a plane Poiseuille flow. We use Brinkman's model to describe the porous medium and investigate the influence of the depth ratio $\hat{d}$ and the Darcy number $\delta$ on the instability of the system. We also compare thoroughly the characteristics of the instability of Brinkman's model with that of Darcy's model. Major results of this study can be summarized as follows.

For Darcy's model, there are three instability modes when the Darcy number $\delta$ is small. When the depth ratio is small, the long wave branch is the most unstable and the porous mode is favored. As $\hat{d}$ increases, the instability switches from the porous mode to the odd-fluid-layer mode. Such an odd-fluid-layer mode exists only in a small range of depth ratio $\hat{d}$. For a more larger $\hat{d}$, the even-fluid-layer mode 
becomes dominant. In our results, we found that when even-fluid-layer mode occurs, the marginal curves are multivalued.

For Brinkman's model, only two instability modes are found in our results, i.e., the porous mode and the even-fluidlayer mode. The odd-fluid-layer mode for Darcy's model will not occur for Brinkman's model. In the low permeability case, the marginal curve displays a bimodal structure and the short wave mode is preferred. With the increase in $\hat{d}$, the system becomes more stable. When the short wave (evenfluid-layer) mode is dominant, the system behaves like the plane Poiseuille flow and the porous medium behaves somewhat like a solid boundary. In the high permeability case, when the depth ratio $\hat{d}$ is small, the porous mode is preferred. With the increase in $\hat{d}$, the short wave branch is present and both the long wave branch and the short wave branch become more unstable. For larger $\hat{d}$, the porous mode is suppressed by the even-fluid-layer mode.

The Darcy number $\delta$ is an important parameter to influence the instability of the system. For small $\delta$, the short wave branch is more unstable than the long wave branch. With the increase in $\delta$, the instability switches a short wave mode to a long wave mode.

The instability of a fluid-porous system predicted by the Brinkman model is different from the results of the Darcy model. The question which model predicts the reasonable results can only be answered by careful experiments.

\section{ACKNOWLEDGMENTS}

The authors are grateful for the financial support for this research from National Foundation of China (Grant Nos. 10772185 and 10372105) and the Knowledge Innovation Program of Chinese Academy of Sciences (Grant No. KGCX-SW-409). We also thank Dr. Bing Hong Zhou for many useful discussions.
${ }^{1}$ A. Davey and P. G. Drazin, "The stability of Poiseuille flow in a pipe," J. Fluid Mech. 36, 209 (1969).

${ }^{2}$ A. Davey and H. P. F. Nguyen, "Finite-amplitude stability of pipe flow," J. Fluid Mech. 45, 701 (1971).

${ }^{3}$ L. H. Thomas, "The stability of plane Poiseuille flow," Phys. Rev. 91, 780 (1953).

${ }^{4}$ C. E. Grosch and H. Salwen, "The stability of steady and time-dependent plane Poiseuille flow," J. Fluid Mech. 34, 177 (1968).

${ }^{5}$ S. A. Orszag, "Accurate solution of the Orr-Sommerfeld stability equation," J. Fluid Mech. 50, 689 (1971).

${ }^{6} \mathrm{~F}$. Chen and C. F. Chen, "Onset of finger convection in a horizontal porous layer underlying a fluid layer," J. Heat Transfer 110, 403 (1988).

${ }^{7}$ B. Straughan, "Surface-tension driven convection in a fluid overlying a porous layer," J. Comput. Phys. 170, 320 (2001).

${ }^{8}$ T. Desaive and G. Lebon, "Coupled capillary and gravity-driven instability in a liquid film overlying a porous layer," Phys. Rev. E 64, 066304 (2001).

${ }^{9}$ M. H. Chang, F. Chen, and B. Straughan, "Instability of Poiseuille flow in a fluid overlying a porous layer," J. Fluid Mech. 564, 287 (2006).

${ }^{10}$ G. S. Beavers and D. D. Joseph, "Boundary conditions at a naturally permeable wall,” J. Fluid Mech. 30, 197 (1967).

${ }^{11}$ M. Le Bars and M. G. Worster, "Interfacial conditions between a pure fluid and a porous medium: Implications for binary alloy solidification," J. Fluid Mech. 550, 149 (2006).

${ }^{12}$ D. A. Nield and A. Bejan, Convection in Porous Media (Springer, New York, 1999).

${ }^{13}$ P. H. Zhao and C. F. Chen, "Stability analysis of double-diffusive convection in superposed fluid and porous layers using a one-equation model," Int. J. Heat Mass Transfer 44, 4625 (2001).

${ }^{14}$ M. Hennenberg, M. Z. Saghir, A. Rednikov, and J. C. Legros, "Porous media and the Bénard-Marangoni problem," Transp. Porous Media 27, 327 (1997).

${ }^{15}$ H. C. Brinkman, "A calculation of the viscous force exerted by a flowing fluid on a dense swarm of particles," Appl. Sci. Res., Sect. A 2, 27 (1947).

${ }^{16}$ P. G. Drazin and W. H. Reid, Hydrodynamic Stability (Cambridge University Press, Cambridge, England, 1981).

${ }^{17}$ M. H. Chang, "Thermal convection in superposed fluid and porous layers subjected to a horizontal plane Couette flow," Phys. Fluids 17, 064106 (2005).

${ }^{18} \mathrm{M}$. H. Chang, "Thermal convection in superposed fluid and porous layers subjected to a plane Poiseuille flow," Phys. Fluids 18, 035104 (2006).

${ }^{19}$ C. Canuto, M. Y. Hussaini, A. Quarteroni, and T. A. Zang, Spectral Method in Fluid Dynamics (Springer-Verlag, New York, 1988).

${ }^{20}$ L. S. Tuckerman, "Divergence-free velocity field in nonperiodic geometries,” J. Comput. Phys. 80, 403 (1989). 